\title{
Anisotropic Bulk Etching of (110) Silicon with High Aspect Ratio
}

\author{
Seong-Hyok Kim, Sang-Hun Lee, Hyung-Taek Lim, Yong-Kweon Kim and Seung-Ki Lee* \\ School of Electrical Engineering, Seoul National University \\ San 56-1 Shilim-dong, Kwanak-gu, Seoul, 151-742, Korea \\ *Department of Electrical Engineering, Dankook University \\ 8, Hannam-dong, Yongsan-gu, Seoul 140-714, Korea
}

\begin{abstract}
Summary
The characteristics of (110) silicon anisotropic bulk etching are studied with varied temperature and concentration of aqueous $\mathrm{KOH}$ solution. The etch rates of (110) and (111) planes increase with increasing temperature but decrease with increasing concentration. The maximum etch rate ratio is above 150 at 45 exact wt. $\%$ and $60^{\circ} \mathrm{C}$ of aqueous $\mathrm{KOH}$ solution. Surface roughness varies greatly with concentration, and the minimum roughness is observed in an aqueous $\mathrm{KOH}$ solution of 41 exact wt. \%. In 41 exact wt. \% aqueous $\mathrm{KOH}$ solution at $65^{\circ} \mathrm{C}$, comb structure have been fabricated which are $8 \mu \mathrm{m}$ wide, $150 \mu \mathrm{m}$ high and separated by $7 \mu \mathrm{m}$ gaps, using a comb mask pattern $10 \mu \mathrm{m}$ wide with $5 \mu \mathrm{m}$ gaps.
\end{abstract}

KEYWORDS : (110) silicon, anisotropic bulk etching, high aspect ratio, comb structure

\section{INTRODUCTION}

Anisotropic etching of silicon has become an important technology in silicon processing. Anisotropic etching of single crystal silicon, resulting from different etch rates in each of its crystallographic planes, has been used to fabricate various structures[1,2,3]. The anisotropic etching of (110) silicon provides useful means for the fabrication of high-aspect-ratio structures with atomically smooth, vertical sidewalls because of high etching selectivity between (111) and (110) planes.

Experimental research on (110) silicon anisotropic etching has been widely performed. D.M. Allen[4], P. Krause[5], and $Y$. Uenishi[6] experimented with holes and narrow groove patterns for (110) silicon etching under certain specific etching conditions, with fixed concentration or temperature. Kishi[7] etched (110) silicon anisotropically with no mask at higher temperature and obtained etch rate, etch rate ratio and surface roughness in various etching condition. K. Sato also studied characteristics of (110) silicon anisotropic etching with hemispherical specimens in aqueous Potassium Hydroxide(KOH) under several different conditions[8,9]. These studies have shown that the anisotropic etching characteristics of (110) silicon vary with concentration, temperature, and shape of the mask pattern. Therefore, there is a need for study of the etching of (110) silicon with various concentrations, temperatures and patterns.

In this paper, the characteristics of (110) silicon anisotropic bulk etching such as etch rates, etch rate ratio, surface roughness and beam length are studied for fabricating comb structure with varying temperatures, concentrations of $\mathrm{KOH}$ aqueous solution and comb dimension. Comb structures with high aspect ratios are fabricated by using conditions that allow high etch rate ratios and smooth etched surface,.

\section{EXPERIMENT}

Using (110) silicon wafer, we deposited $1000 \AA$ thermal oxide to buffer internal stresses between bulk silicon and $\mathrm{Si}_{3} \mathrm{~N}_{4}$ layer, and $3000 \AA$ LPCVD nitride for a masking layer, in $\mathrm{KOH}$ aqueous solution. (110) wafers have a primary flat to indicate the $\langle 111\rangle$ direction, but this is accurate to only about $\pm 1^{\circ}$ along the exact $<111\rangle$ direction. To find the exact $\langle 111\rangle$ direction more exactly, we used a fan-shaped alignment target composed of $8 \mu \mathrm{m}$ beams, which is then placed $0.1^{\circ}$ between $\pm 4^{\circ}$ range, and the misalignment between an etch mask and the $\langle 111\rangle$ direction was determined to $\pm 0.05^{\circ}$ [10]. By aligning comb pattern along this exact $\langle 111\rangle$ direction, a high-aspect-ratio comb structure can be obtained.

The comb shown in Figure $2,10 \mu \mathrm{m}$ and $20 \mu \mathrm{m}$ wide, respectively and with $5 \mu \mathrm{m}$ gaps, were fabricated to 
investigate the etching characteristics. Etching of (110) silicon is performed at temperatures from $60^{\circ} \mathrm{C}$ to $90^{\circ} \mathrm{C}$ and concentrations of $\mathrm{KOH}$ aqueous solution between 32 exact wt.\% and 45 exact wt.\%. Exact wt.\% is measured by means of neutral titration and the temperature and concentrations are controlled to under $0.5 \%$.

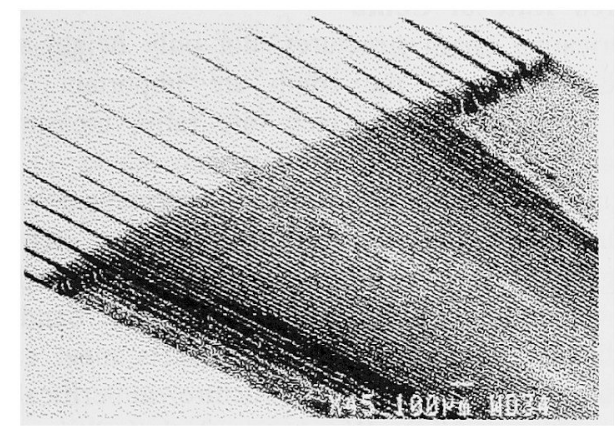

Figure 1. SEM photograph of alignment target after wet etching

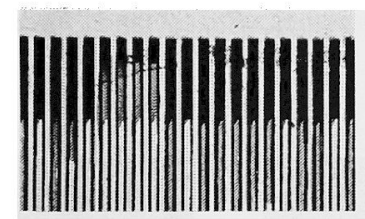

(a) $10 \mu \mathrm{m}$ width, $5 \mu \mathrm{m}$ gap Figure 2. Fabricated width

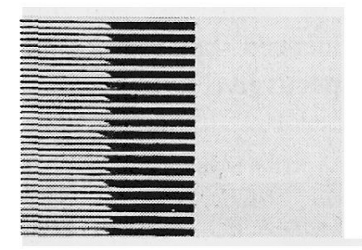

(b) $20 \mu \mathrm{m}$ width, $5 \mu \mathrm{m}$ gap structures with a different

\section{EXPERIMENTAL RESULTS}

\subsection{Etch Rates of (110) and (111) Plane}

Figure 3 and Figure 4 show the etch rate after 100 minutes of (110) plane and (111) plane when (110) silicon bulk etching is performed.

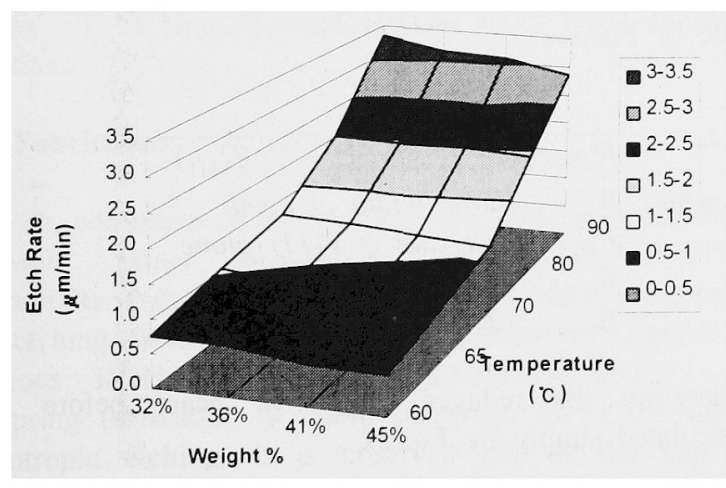

Figure 3. Etch rate of (110) plane

Etch rates of the (110) and (111) planes are calculated by measuring the height and width of the structure using a laser profiler (KEYENCE VF-7500). The etch rates of both planes increase with increasing temperature, and decrease with increasing concentration of $\mathrm{KOH}$ aqueous solution. The temperature of etchant has a strong influence on the relative etch rates of (110) and (111) planes than does concentration of $\mathrm{KOH}$ aqueous solution. Figure 5 shows the ratios of etch rates between (110) and (111) planes.

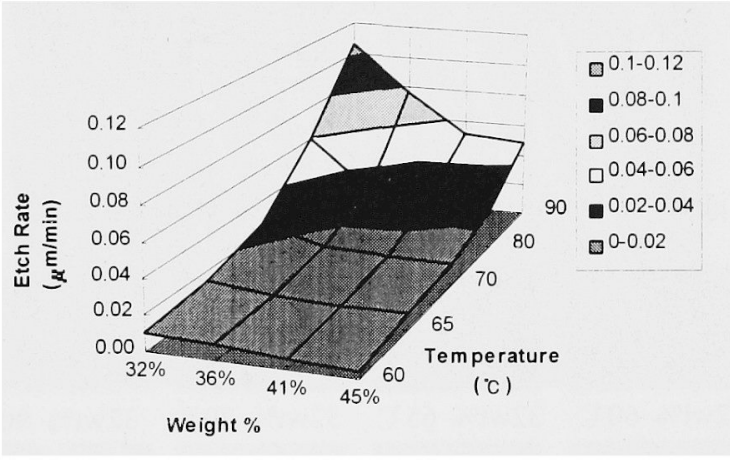

Figure 4. Etch rate of (111) plane

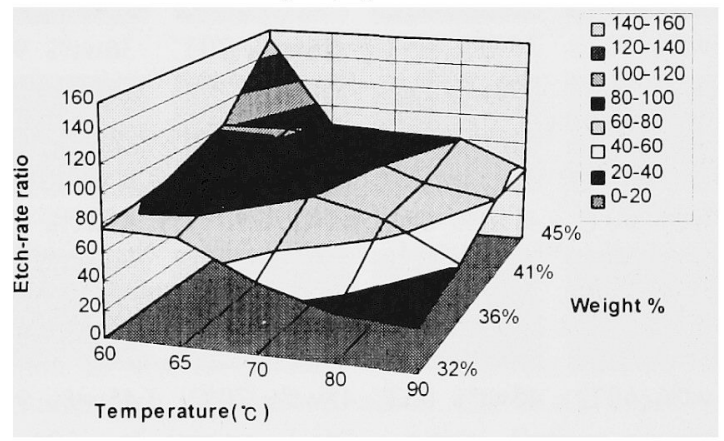

Figure 5. Etch rate ratios between $\mathrm{R}<110>$ and $\mathrm{R}<111>$

The etch rate ratio increases with either increasing $\mathrm{KOH}$ concentration or decreasing temperature. High etch rate ratios, over 100, are obtained in aqueous solutions of 41 and 45 exact wt.\% $\mathrm{KOH}$, at controlled temperature from $60^{\circ} \mathrm{C}$ to $70^{\circ} \mathrm{C}$. The highest etch rate ratio, larger than 150 , is observed in the case of 45 exact wt. $\% \mathrm{KOH}$ aqueous solution at $60^{\circ} \mathrm{C}$.

\subsection{Surface Roughness of Etched Silicon}

The roughness of an etched silicon surface is also important to special applications such as micro mechanical structures or micro optics. They are measured using a laser profiler to study etched surfaces in various conditions of aqueous $\mathrm{KOH}$ solution. Figure 6 shows the surface roughness of etched (110) silicon planes with different depth from 100 to $300 \mu \mathrm{m}$ after 100 minutes etching. The surface roughness was observed to have a minimum at 42 exact wt. $\%$ and a maximum at 
36 exact wt. $\%$ of $\mathrm{KOH}$ aqueous solution.

Figure 7 shows the photographs of (110) etched planes at different temperatures and concentrations of $\mathrm{KOH}$ aqueous solution.

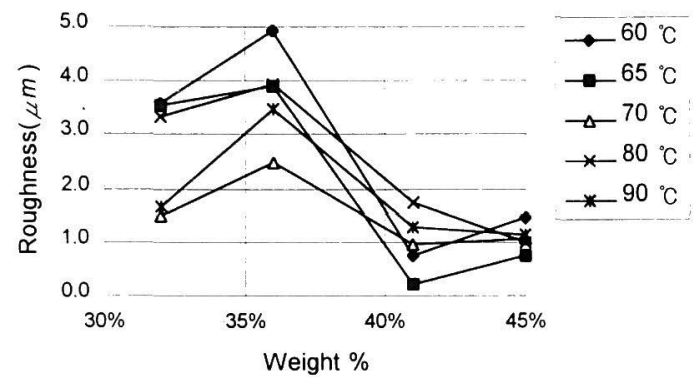

Figure 6. Surface roughness of (110) etched plane

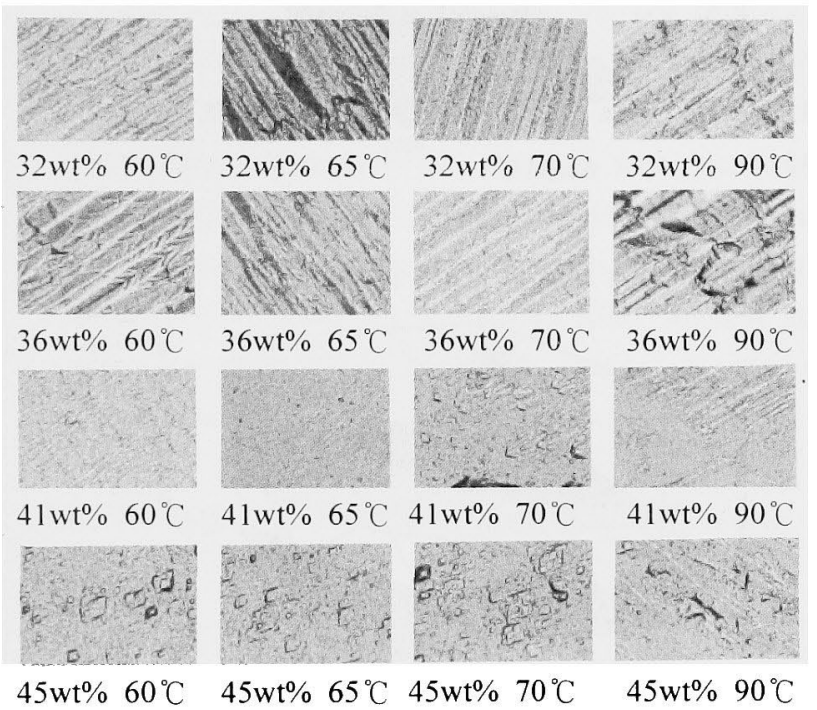

Figure 7. Photographs of (110) etched planes

Characteristic shapes of etched (110) planes are observed at different $\mathrm{KOH}$ concentration. Below 36 exact $w t \%$, etched planes of wave shape are observed, but above 36 exact $w t \%$, hillocks are observed on the (110) etched surface. The surface roughness is larger at high temperatures of etchant.

In all cases, (111) planes are smoother than other planes in SEM photographs as shown in Figure 8.

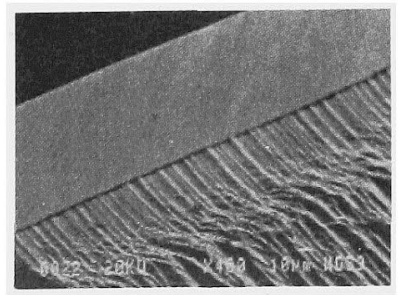

$32 \mathrm{wt} \% \quad 60^{\circ} \mathrm{C}$

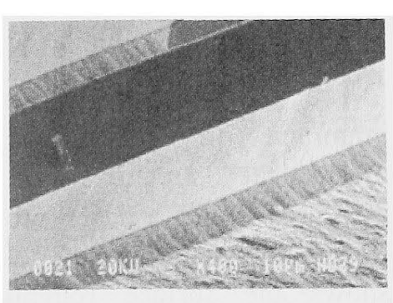

$45 w t \% \quad 65^{\circ} \mathrm{C}$
Figure 8. SEM photographs of (110) and (111) plane after etching
The vertical walls in Figure 8 are (111) plane, and the horizontal surfaces are (110) planes. The figures show that the surface smoothness of the (111) plane is not concerned with the roughness of (110) plane.

\subsection{Etch Rate of Comb}

In fabricating comb structures, not only height and width but also length of the comb are important. Since the length is determined by etch rate ratio, measurement of the etched beam length is required.

Figure 9 shows the etching mechanisms of beam structure. Performing the (110) Silicon etching with rectangular mask, the convex corners are etched to form two fast etched planes of Facet l and Facet immediately(Figure 9(a)), the two facets meet each other to make triangular etched structure at critical etching time $t_{0}$ (Figure 9(b)). We assumed that during the step shown in Figure 9(a), the Facet $_{m}$ which is perpendicular to the (111) plane has strong influence on the etch rate of beam length. However, in next step, this facet seems to be vanished, and the etch rate of beam length is influenced by two fast etch planes Facet, and Facet, respectively(Figure 9(c))

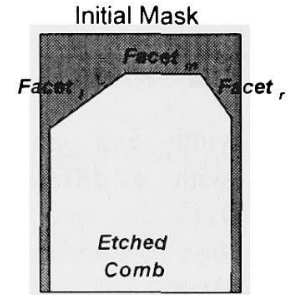

(a) $t<t_{0}$

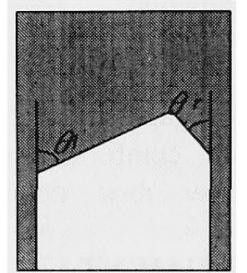

(b) $t=t_{o}$

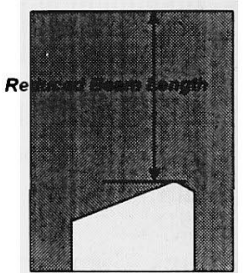

(c) $t>t_{0}$
Figure 9. Etching mechanism of beam structure

Critical etching time $t_{0}$ from (a) to (c) can be determined by etch rates of Facet, and Facet $t_{r}$ as in Eq.(1) and the etch rate of beam is equal to the etch rate of Facet $_{m}$.

$$
\begin{aligned}
& t_{0}=\frac{W}{\left(R_{l} \sec \theta_{l}+R_{r} \sec \theta_{r}+2 \times R_{111}\right)} \\
& \text { where } W \text { : Initial width of comb } \\
& R_{111} \text { : Etch rate of (111) plane } \\
& R_{l} \quad \text { : Etch rate of Facet }{ }_{l} \\
& R_{r} \quad \text { : Etch rate of Facet }{ }_{r}
\end{aligned}
$$

Therefore the reduced length of beam before $t_{0}$ is calculated simply by Eq.(2).

$$
\begin{aligned}
& L_{\text {Reduced }}=R_{m} \times t \\
& \text { where } R_{m}: \text { Etch rate of Facet } \text { F }_{m} \\
& t \quad: \text { etching time }\left(<t_{0}\right)
\end{aligned}
$$


After two facets Facet, and Facet r meet each other as shown in Figuire 9(b), the remained beam length can be expressed as Eq.(3) and simplified etch rate of beam can be calculated simply as Eq.(4).

$$
\begin{aligned}
L_{\text {Reduced }} & =L_{t_{0}}+R_{\text {Beam }}\left(t-t_{0}\right) \\
R_{\text {Beam }}= & R_{l p}-\frac{\cot \theta_{l}}{\cot \theta_{l}+\cot \theta_{r}}\left(R_{l p}-R_{r t}\right. \\
& \left.-2 \times \cot \theta_{r} \times R_{111}\right)
\end{aligned}
$$

where $\mathrm{L}_{t 0}$ is the length of beam at the time $t_{0}$ $R_{\text {Beam }}$ is the etch rate of beam $R_{l p}=R_{m}+R_{l} \operatorname{cosec} \theta_{l}$ $R_{r p}=R_{m}+R_{r} \operatorname{cosec} \theta_{r}$ and $R_{l p}, R_{r p}$ are lengthwise etch rate of beam

Figure 10 shows that the measured length of beam coincides with the calculated value, and the difference of beam length results from the initial etching time which is dependent on the width of comb. However, after initial etching, beams are linearly decreased independent on the comb width. Consequently, as the width of the beam is wider, the remained beam is longer.

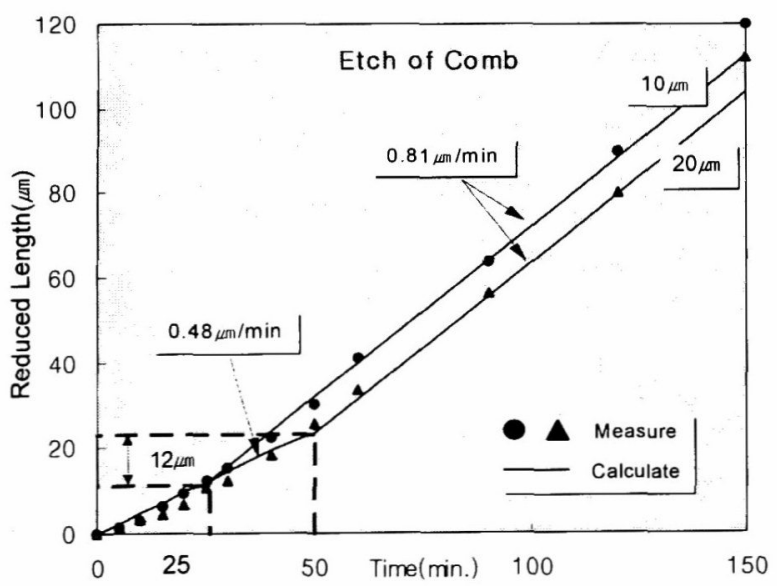

Figure 10. Etching of beam $\left(41 \mathrm{wt} . \%, 65^{\circ} \mathrm{C} \mathrm{KOH}\right.$ aqueous solution)

\subsection{Fabrication of Comb Structure}

With consideration of etching condition such as high etch-rate ratios between $\mathrm{R}<110>$ and $\mathrm{R}<111>$, appropriate etch time, and minimum surface roughness, the etching condition for comb structures was chosen as aqueous $\mathrm{KOH}$ solution of 41 exact $\mathrm{wt} \%$ at $65^{\circ} \mathrm{C}$.

During fabrication of comb structures by (110) silicon anisotropic etching, it is observed that if the gap of comb is narrow, then the etch rate of narrow gap is smaller than that of widely opened surfaces as Ohwada reported[11]. The etched depth of narrow gap is about $30 \mu \mathrm{m}$ shallower than that of the wider one, therefore 30 minutes overetching can make these structures wholly penetrated.

Using this etching condition, we successfully fabricated comb structures which are $8 \mu \mathrm{m}$ wide, $150 \mu \mathrm{m}$ high and with $7 \mu \mathrm{m}$ gaps using a comb mask pattern of $10 \mu \mathrm{m}$ width and $5 \mu \mathrm{m}$ gap(Figure 11.)

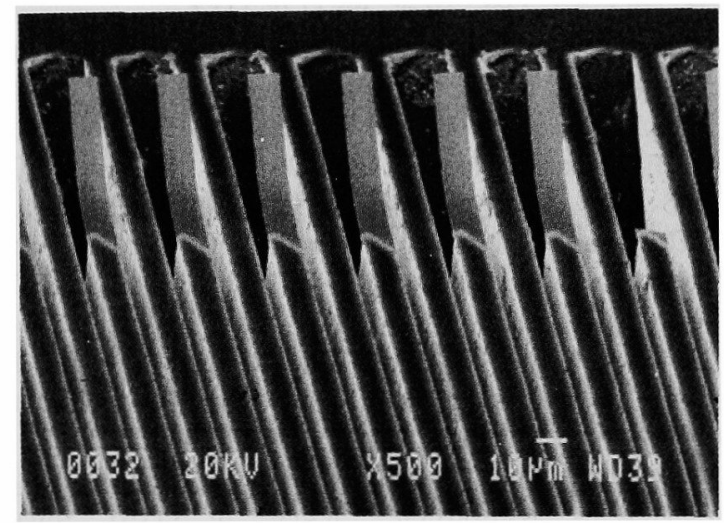

Figure 11. Fabricated comb structures. The width is $8 \mu \mathrm{m}$, gap is $7 \mu \mathrm{m}$ and height is about $150 \mu \mathrm{m}$

\section{CONCLUSION}

We have studied the characteristics of (110) silicon anisotropic etching with varying temperature and concentration of aqueous $\mathrm{KOH}$ solution. The etch rates of (110) and (111) planes increase with temperature increasing and decrease with the concentration decreasing. High etch rate ratios are observed in $\mathrm{KOH}$ solution at low temperature and high concentration. The maximum etch rate ratio is above 150 at 45 exact $w t . \%$ and $60^{\circ} \mathrm{C}$ of aqueous $\mathrm{KOH}$ solution. Surface roughness varies greatly with concentration of etchant, and increases with rising temperature. The maximum surface roughness is observed in 36 exact wt.\% and the minimum surface roughness in 41 exact wt. $\%$ of aqueous $\mathrm{KOH}$ solution.

To fabricate comb structure of high-aspect-ratio and smooth surface, we chose 41 exact wt. $\% \mathrm{KOH}$ solution at $65^{\circ} \mathrm{C}$. In these conditions, comb structures have been fabricated $8 \mu \mathrm{m}$ wide, with $7 \mu \mathrm{m}$ gaps and $150 \mu \mathrm{m} \mathrm{high,}$ using a comb-shaped mask pattern with lines $10 \mu \mathrm{m}$ wide and $5 \mu \mathrm{m}$ apart.

\section{ACKNOWLEDGEMENTS}

This work has been supported by the Ministry of Education and Inter-University Semiconductor Center (ISRC 96-E-5011) in Korea.

(Manuscript received April 3, 1997, revised Aug. 14, 1997) 


\section{REFERENCES}

[1] E.Bassous, "Fabrication of Novel Three-Dimensional Microstructures by the Anisotropic etching of (100) and (110) Silicon," IEEE Trans. Electron Devices, Vol. ED-25, NO. 10, Oct. 1978, pp.1178-1184

[2] D.L.Kendall, "Vertical etching of silicon at very high aspect ratios," Ann. Rev. Mater. Sci. 1979.9, pp.373-403

[3] K.E.Bean, "Anisotropic Etching of Silicon," IEEE Trans. Electron Devices, Vol.Ed-25, No. 10, Oct. 1978, pp.1185-1193

[4] D.M.Allen, "Anisotropic Etching of Silicon:a Model Diffusion Controlled Reaction," IEE Proc., Vol. 130, No. 2, 1983, pp.49-56

[5] P.Krause, "Etch rate and surface roughness of deep narrow U-grooves in (110)-oriented silicon," J.Micromech. Microeng. 5, 1995, pp.112-114

[6] Y.Uenishi, "Micro-Opto-Mechanical Devices Fabricated by Anisotropic Etching of (110) Silicon," IEEE MEMS, 1994, pp.319-324

[7] H.Kishi, "Micropyramid Inhibition Method in Anisotropic Etching of Silicon," Shinkuu, Vol. 29, No. 2, 1986, pp.85-91

[8] A.Koide, K.Sato, "Simulation of two-dimensional etch profile of silicon during orientation-dependent anisotropic etching," IEEE MEMS, 1991, pp.216-220

[9] K.Sato, "Characterization of anisotropic etching properties of single-crystal silicon:effects of $\mathrm{KOH}$ concentration on etching profiles," IEEE MEMS, 1997, pp. 406-411

[10] D.Zielke, "Determination of rates for orientation-dependent etching", Sensors and Actuators A 48, 1995, pp.151-156

[11] K.Ohwada, "Groove depth Uniformization in (110) $\mathrm{Si}$ Anisotropic Etching by Ultrasonic Wave and Application to Accelerometer Fabrication," Sensors and Actuators A 50, 1995, pp.93-98

\section{BIOGRAPHIES}

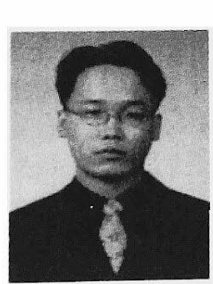

Seong-Hyok Kim(Non-Member) $\mathrm{He}$ received the B.S degree in electrical engineering from Seoul National University, Korea in 1996. $\mathrm{He}$ is currently for his M.S. degrees at the Laboratory for Micro Sensors and Actuators, School of Electrical Engineering, Seoul National University,

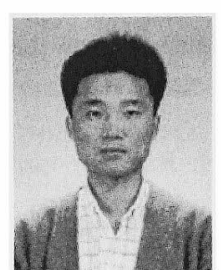

Sang-Hun Lee(Non-Member) $\mathrm{He}$ received the B.S. and M.S. degrees in electrical engineering from Seoul National University, Korea, in 1991 and 1993, respectively. $\mathrm{He}$ is currently for his Ph.D degrees at the Laboratory for Micro Sensors and Actuators, School of Electrical Engineering, Seoul National University, Korea.

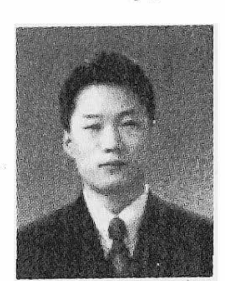

Hyung-Taek Lim(Non-Member) He received the B.S. and M.S. degrees in electrical engineering from Seoul National University, Korea, in 1994 and 1997, respectively. $\mathrm{He}$ is currently for his Ph.D degrees at the Laboratory for Micro Sensors and Actuators, School of Electrical Engineering, Seoul National University, Korea.

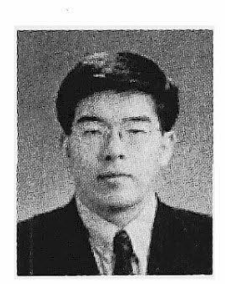

Yong-Kweon Kim(Member) He received the B.S. and M.S. degrees in electrical engineering from Seoul National University, Korea, in 1983 and 1985, respectively, and the Dr. Eng. degree from the University of Tokyo in 1990. In 1990, he joined the Central Research Laboratory of Hitachi Ltd. in Tokyo as a researcher and worked on actuators of hard disk drives. In 1992, he joined Seoul National University, where he is currently an Associate Professor in School of Electrical Engineering.

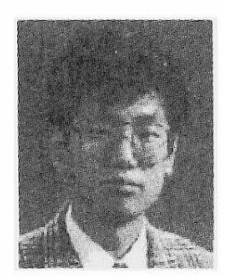

Seung-Ki Lee(Non-Member) He received the B.S. and M.S. degrees in electrical engineering from Seoul National University, Korea, in 1986 and 1988, respectively, and his Ph.D. degree from the Department of Electrical Engineering of Seoul National University in 1992. From 1992 to 1993, he was at Tohoku University, Japan, as a guest researcher with a JSPS fellowship, where he conducted research on microactuators. In 1994, he joined the Department of Electrical Engineering, Dankook University, where he is now an assistant professor. He is a member of the IEEE and the KIEE.

Korea. 